\title{
Type Ia Supernovae as Probes of the Interstellar Medium in Galaxies
}

\author{
N. L. J. $\operatorname{Cox}^{1}$ and F. Patat ${ }^{2}$ \\ ${ }^{1}$ Instituut voor Sterrenkunde, KU Leuven, Celestijnenlaan 200D, 3001 Leuven, Belgium \\ email: nick@ster.kuleuven. be \\ ${ }^{2}$ ESO, Garching, Germany
}

\begin{abstract}
We present and discuss recent results from observations of bright Type Ia supernovae as probes of physical and chemical condtions of extra-galactic interstellar clouds. To illustrate the case we present new results obtained for SN 2009ig.
\end{abstract}

Keywords. ISM: lines and bands, dust, extinction, ISM: clouds, ISM: structure, supernovae: 2009ig

\section{The early years (Introduction)}

Width the advent of modern-day astronomical instrumentation and large telescopes supernovae have been used as external light beacons to probe the interstellar medium of nearby galaxies in the Local Group, Centaurus A/M83 group and the Virgo Cluster. In the 1980s several bright nearby Type Ia supernovae (SNe) were studied at moderate (10$20 \mathrm{~km} \mathrm{~s}^{-1}$ ) spectral resolution: SN 1983N in (Jenkins et al. 1984, D'Odorico, Pettini \& Ponz 1985), SN 1986G (Phillips et al. 1987, Rich 1987, D'Odorico et al. 1989, SN 1987A (Vidal-Madjar et al. 1987, Vladilo et al. 1987), and SN 1989M (Steidel et al. 1990). These studies provided direct detection and measurement of both interstellar atomic and molecular lines, as well as diffuse interstellar bands. In particular SN 1987 due to its proximity - it exploded in the Large Magellanic Cloud, our nearest neighbour galaxy - allowed for detaild high-resolution ( $\mathrm{R} \sim 100000$ or $\sim 3 \mathrm{~km} \mathrm{~s}^{-1}$ ) studies of the ISM that were later followed-up in detail when 8-10 $\mathrm{m}$ class telescopes allowed for individual line-ofsight studies towards early-type stars in the Magellanic Clouds and other Local Group galaxies (Cordiner, this volume; Smith; this volume). SNe offer, next to quasars and gammar-ray burst afterglows, one of few oportunities to study extra-galactic interstellar medium beyond the Local Group. The contribution briefly discusses what has been achieved with SNe in the context of ISM studies as well as their potential usefulness to other fields.

\section{Multi-epoch monitoring of Type Ia supernovae: Taking advantage for ISM studies}

In recent years the study of extra-galactic ISM has especially benefitted from the growing interest in high-resolution multi-epoch monitoring campaigns of Type Ia supernovae that aim to detect small variations in e.g. sodium and potassium abundances in the circumstellar environment of Type Ia supernovae as those may help to constrain their progenitor systems (Patat et al. 2007). These observations, in many cases obtained with UVES at the Very Large Telescope, have shown that DIB carriers and carbon molecules are abundant also beyond the Local Group, including the Milky Way, but also in the 


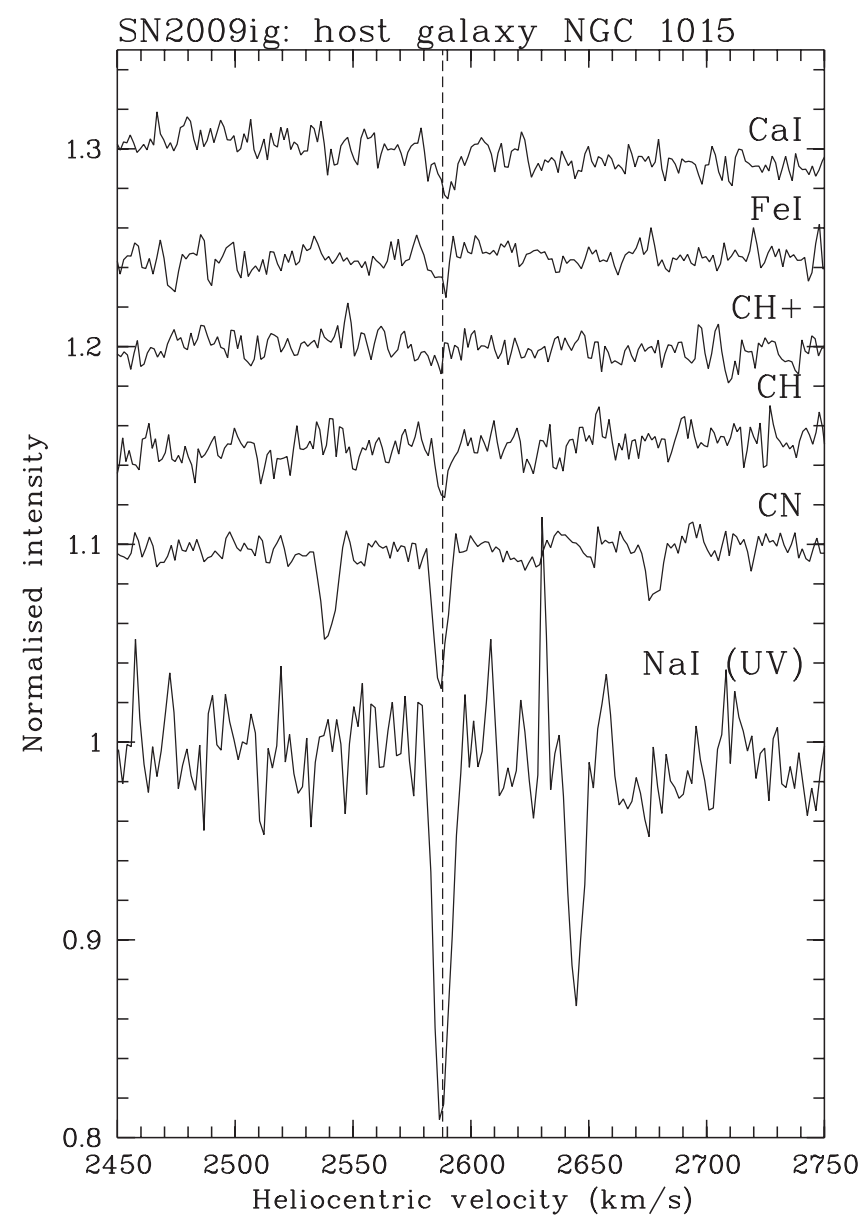

Figure 1. Interstellar absorption lines of atoms and di-atomic molecules towards SN 2009ig plotted on a heliocentric velocity scale. These spectra constitute an average over three epochs for a total exposure time of 5.5 hours with UVES at the VLT achieving a S/N of 50 for the $\mathrm{Na}$ I UV doublet and $\mathrm{S} / \mathrm{N}$ between 150 and 200 for $\mathrm{CN}, \mathrm{CH}, \mathrm{CH}^{+}, \mathrm{Fe}$, and Ca I. We also investigated the spectra for the presence of interstellar Ti II, Al I, and K I but these transitions were not detected. The systemic host galaxy velocity is $2629 \mathrm{~km} \mathrm{~s}^{-1}$. The strongest interstellar component is observed at $2588 \mathrm{~km} \mathrm{~s}^{-1}$ (vertical dashed line).

interstellar medium of more distant $(d \geqslant 20 \mathrm{Mpc})$ spiral galaxies (e.g. Sollerman et al. 2005, Cox \& Patat 2008, Thöne et al. 2009, Patat et al. 2013).

\section{The "Diffuse Interstellar Band" Toolbox}

Apart from the treasure trove these recent observations form to study in great detail the physics and chemistry of extra-galactic interstellar medium, measurements of DIBs are now also employed to infer line-of-sight properties that are otherwise difficult to measure. In particular the line-of-sight extinction can be reasonable well predicted (within roughly a factor of two) from several individual strong DIBs that correlated well (not perfectly!) with reddening, E(B-V), as determined for Galactic lines-of-sight. With sufficiently accurate measurements of DIBs and serveral di-atomic molecular species, other environmental paramters can also be estimated, such as the radiation field strength and the molecular 


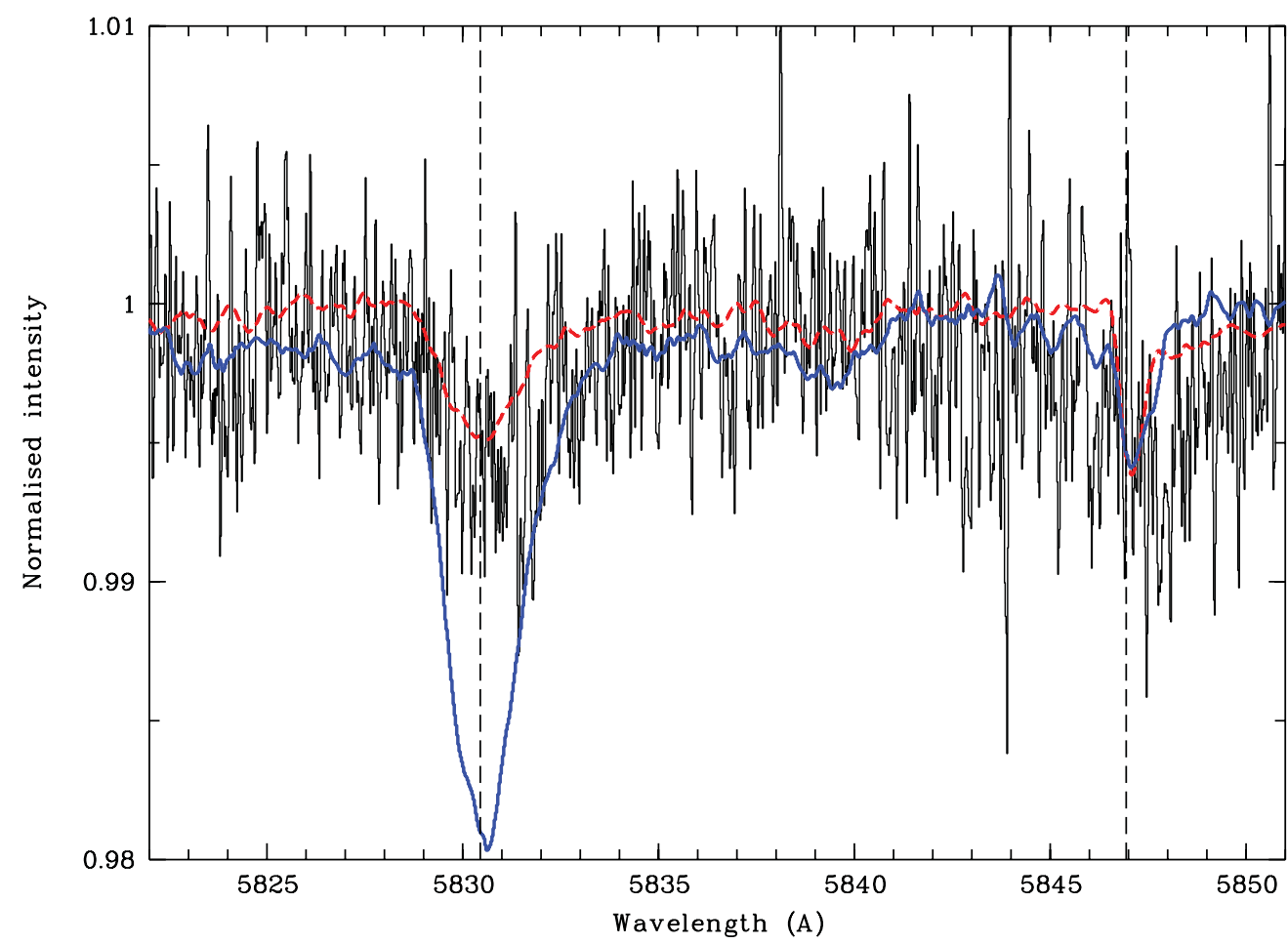

Figure 2. Tentative detection of 5780 and 5797 DIBs towards SN 2009ig. Continuum S/N is $\sim 200$. The vertical dashed line indicates the radial velocity corresponding to the strongest atomic and molecular lines arising from the ISM in NGC 1015 (see Fig. 1). The thick solid blue line represents the DIBs observed towards HD 144217 ( $\sigma$ Sco) whose line-of-sight probes a so-called $\sigma$-type single cloud. The thick dashed red line shows the DIB spectrum towards HD 149757 $(\zeta \mathrm{Oph})$, whose line-of-sight probes a so-called $\zeta$-type cloud. (Krełowski, this volume) Both absorption spectra are scaled in intensity to correspond to $\mathrm{E}(\mathrm{B}-\mathrm{V})=0.05$ mag. $\sigma$-type clouds are highly exposed to the interstellar radiation field, while $\zeta$-type clouds constitute more UV protected environments (see e.g. Vos et al. 2011). $\zeta$-type environments are also known to exhibit much stronger $\mathrm{CN}$ transitions per unit reddening compared to $\sigma$-type clouds (Krełowski, this volume). Similar to the comparision for DIBs towards SN 2001el (Sollerman et al. 2005) we show here that the ISM in NGC 10515 towards SN 2009ig is reminiscent to $\zeta$-type environment, but suffering little dust extinction, with $\mathrm{E}(\mathrm{B}-\mathrm{V})$ of 0.06 mag.

hydrogen fraction (Vos et al. 2011). Thus, with some prudency the strongest diffuse interstellar bands may be used to obtain independent measurements of the SN host galaxy or Galactic foreground line-of-sight dust extinction which both affect the observed SN light curve.

Similarly, Phillips et al. (2013) use the 5780 DIB detected in 11 SN host galaxies to estimate visual extinction and infer an overabundance of $\mathrm{Na}$ I for several Type Ia supernovae. In addition to atomic and di-atomic species it is now also possible to directly detected absorption from the smallest carbon chain, $\mathrm{C}_{3}$, beyond the Local Group (see e.g. Cox \& Patat 2014). Also, CN lines can be used to probe the cosmic microwave background (CMB) temperature in external galaxies. To illustrate the quality and richness of the data we present in this contribution unpublished VLT/UVES spectra $(\mathrm{R} \sim 80000)$ obtained for SN 2009ig (Figures 1 and 2). 


\section{Conclusions}

The hithertho collected set of observations have primarily revealed that diffuse band line profiles in this extra-galactic environments are similar to those for local lines-of-sight, and their relative intensities can, together with the optical transitions of $\mathrm{CH}, \mathrm{CH}^{+}$, and $\mathrm{CN}$, be used as probes of the local ISM. With new instrumentation and facilities it will become possible to study in more-and-more detail the chemical and physical conditions of the interstellar mediuim in distant galaxies. Hence this in turn will give important insights into the abundance and distribution of the DIB carriers over cosmic time which will benefit both the extra-galactic community as well as the search for the carrier identity.

\section{References}

Cordiner, M. A. 2013, Proceedings IAU symposium 297, this volume

Cox, N. L. J. \& Patat, F., 2008, A\&A Letters, 485, 9

-. 2014, $A \& A$, in press

D’Odorico, S. Pettini, M., \& Ponz, D. 1985, ApJ, 299, 852

D’Odorico, S., di Serego Alighieri, S., Pettini, M., et al. 1989, A\&A, 215, 21

Jenkins, E. B., Rodgers, A. W., Harding, P., Morton, D. C., \& York, D. G. 1984, ApJ, 281, 585

Krełowski, Proceedings IAU symposium 297, this volume

Patat, F. 2007, Science, 317, 924

Patat, F., Cordiner, M. A., Cox, N. L. J., et al. 2013, A\&A A, 549, A62.

Phillips, M. M., Phillips, A. C., Heathcote, S. R., et al. 1987, PASP, 99, 592

Phillips, M. M., Simon, J. D., Morrell, N., et al. 2013, ApJ, 779, 38

Rich, R. M. 1987, AJ, 94, 651

Smith, K. T. 2013, Proceedings IAU symposium 297, this volume

Sollerman, J., Cox, N. L. J., Matilla, S., et al. 2005, A\&\&A, 429, 559

Steidel, C. C., Rich, R. M., \& McCarthy, J. K. 1990, AJ, 99, 1476

Thöne, C. C., Michalowski, M. J., Leloudas, G., Cox, N. L. J., et al. 2009, ApJ, 698, 1307

Vidal-Madjar, A., Andreani, P., Cristiani, S., et al. 1987, A\& $A$ Letters, 177, 17

Vladilo, G., Crivellari, L., Molaro, P., \& Beckman, J. E. 1987, A\&A Letters, 182, 59

Vos, D., Cox, N., Kaper, L., Spaans, M., \& Ehrenfreund, P. 2011, A\&̛A, 533, A129 